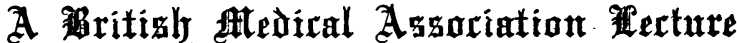
ON

\section{THE TREATMENT OF BURNS IN CHILDREN.}

Grven to the Aberdeen Division, April 21st, 1927, BY

JOHN FRASER, M.C., M.D., F.R.C.S.ED.,

REGIUS PROFESSOR OF CLINICAL SURGERY IN THE UNIVERSITY OF EDINBURGH.

"THE child is burned!" I never hear that announcement without a shudder, for it has opened the portals to a long avenue of pain and distress, an avenue that may lead to an age-long disfigurement and too often, by shorter ways, to the tragedy of death. Let us take counsel together about it. Wherein are its dangers? What are its risks? How can disaster be mitigated or avoided?

Some years ago I took the opportunity of investigating one particular aspect of the problem-it was that of mortality; the result of the investigation was disconcerting. In a total of 200 cases of burns affecting children up to the twelfth year the death rate averaged 30 per cent. I know it is a startling figure. It is true that the cases from which the records were culled were hospital cases, and so generally of a serious type; it is, moreover, correct to say that the sufferers were children, and therefore more likely to show a heavy mortality. About these facts there is no dispute, and yet I am distressed when $I$ recall that some few years ago one child in three suffering from a burn of moderate severity perished. I have with intent used the term " some few years ago," because recent improvements in the methods of treatment. have done much to lighten the shadow. It is of these improvements and advances that I would speak.

The Medico-Legal Aspect.

The subject has a medico-legal aspect, and, as it is one sometimes forgotten, I refer briefly to it.

The statute book contains a law that it is an offence to leave a child of tender years alone in a room with an open fire. The provision is a wise one, and no doubt it has done much to diminish the incidence of burns; but something more is required, for ways and means of escaping the penalties are not difficult to imagine. Certain hospitals recognize the deficiencies of the existing statute, and have instituted regulations which make all cases of burns admitted to their charge notifiable incidents. The notification is to the police, and they, having made the inquiries they consider necessary, take whatever further steps they deem advisable. Experience shows that this later measure is one of real efficacy, and it is to be hoped that its application may be made universal.

\section{Causes.}

There are, of course, a multitude of ways in which burns may be caused, but, where children are concerned, it is the pulling over of the pot of hot tea or boiling water, and the accidental ignition of the clothing, which are the principal causes of the disaster. In both instances there is the one common and significant feature that the burn is likely to be of considerable surface extent, without, it may be, any real depth of destruction-a feature with considerable bearing upon the ultimate issue.

There are many other points of significance in this matter of the origin of burns to which I need not allude-the daily press disseminates the knowledge admirably: the danger of flannelette as a garment; the education of the child to avoid fire in any shape or form; the demonstration that, when all is said and done, "it was curiosity that once upon a time killed the cat." These are all very minor matters, it may be, and yet how often a young life has been the price paid for their neglect!

\section{Pathologr.}

Textbooks, with the dogmatism that is expected from them, have classified burns into various stages or degrees according to the extent. of tissue destruction. Such an arrangement no doubt serves its purpose, but it need not concern us to-day. It is rather of the general facts of pathology that I would speak.

When a burn is sustained, a certain amount of cellular disintegration inevitably ensues, and, according to the degree of heat applied, the process varies from complete charring and carbonization to coagulation effects upon the protoplasm of the cell, or it may be to simple reactive phenomena in the cells and blood vessels. This is the significant point-carbonization is not a feature of real importance where the subsequent pathology is concerned. It is certainly an irreparable loss of what may be highly important tissues, but so far as any harmful effects upon the biochemistry of the organism are concerned, the process of destruction has been so complete that the part affected is now an inorganic and inert product, and harmless as far as any dangerous influence is concerned.

It is vastly different, however, with those tissues in which the process of destruction has been less complete. Coagulation of the protoplasm is evidently a process fraught with considerable danger. For a certain period after the injury biochemical activity is in abeyance, but thereafter products of intense toxicity are liberated, which, being absorbed into active circulation, and evidently being peculiarly inimical to the specialized parenchyma cells of the Iiver, kidney, lymphoid tissue, and tho central nervous system, originate a group of serious and distinctive symptoms. These facts have been shown by the experimental work of Willis, Vogt, and other observers, but we are apt to forget them and the influence which they exert in the ultimate clinical picture. I shall have occasion later to allude more fully to the general picture and its relations to clinical features, prognosis, and treatment.

The pathology of a burn is thus something far beyond tho local lesion. Widespread chemical changes, many of them imperfectly understood, ensue, and what was at first a local tissue destruction ultimately becomes the picture of a body chemistry distorted and a metabolism deranged.

The ultimate end of the story is characteristic: the body alkalis become depleted; the buffer salts, the last line of defence in so many " tight corners," have yielded up their resources; the sufferer passes in a condition in which the hydrogen-ion content of the body fluids is increased, and the condition we speak of clinically as " acidosis" ensues.

The Curntcal Picture.

The majority of burns in children are extensive in area and superficial in degree, as one would expect from the rapidly extending flow of steam or boiling water, or the spreading conflagration of the burning garments. A certain measure of shock is always present, and its degree is variable; in some instances it is so severe that a fatal issue is a matter of hours, in other cases it is so slight as to pass unnoticed. Its measure depends upon such various features as the age of the child, the position and extent of the burn, and the psychology of the individual. The dry heat of the flame is associated with a greater measure of shock than the moist heat of the steam or water.

The immediate local effect of a burn is temporarily anaesthetic, but after a few minutes there is intense suffering. The pain is probably relieved by hypnotics, and it would seem that with the passing of the initial shock the most dangerous phase is over, and that the issue, though no doubt a long and a painful process, is not likely to be in doubt as regards life.

For one or two days the optimism appears to be justified, and it may be the case progresses to a favourable issue, but in many instances the progress is otherwise. About the third day the temperature, hitherto keeping within normal limits, or being slightly elevated after the " shock" fall, rises to a level of $101^{\circ}$ or $102^{\circ}$. There is an ominous restlessness, the pulse rate quickens, and-most suggestive of all-the breathing alters, at first as shown by an increase iil rate, and later as a hyperpnoea from an abnormal stimulus of the respiratory centre. The restlessness passes into delirium, and this in a fatal case is followed by coma. The rapid pulse is a prelude to a developing cardiac weakness, and more general evidences are seen in the slight general cyanosis and the cold extremities.

I imagine there are few of you with experience of burns 
who have not seen this tragic sequence of events, and the development is all the more distressing because it is unlooked for, so that a favourable prognosis may have been given after the danger of the initial shock had passed away.

\section{The Correlation of the Pathology and the Chinicat} Features.

The point in the clinical history peculiarly critical is about the third day, when the temperature rises and signs of intoxication become apparent. An indication has already been given of the explanation of the event, but it is not out of place at this stage to elaborate the reasoning more fully.

It is the existence within the body of tissues in a state of chemical dissolution that is evidently the exciting feature of the disturbance-not an organismal decomposition, but a chemical change, and one, moreover, which results in the production of potent toxic elements. We have set to learn the true nature of the product or products. It has been suggested that they are closely allied with, if not exactly similar to, histamine or pyridine. Certainly there are many points of similarity between the picture of " histamine shock" and that of the toxic stage of burns. The dangerous character of the toxaemia depends upon its liability to pick out and deteriorate specialized tissuesthe liver cells, the delicate cells of the kidney tubules, and the chromaffin tissue of the suprarenals. The striated heart muscle is poisoned and degenerated, and the lymphoid tissue shows points of focal necrosis. It is not difficult to explain the symptoms and signs which distinguish the early stages of the toxic phase-the rise of temperature, the restlessness, the rapid pulse-each has its pathological counterpart, but, underlying all this, there is some more subtle influence which specifically and definitely deranges metabolic processes. I agree that no individual substance has been isolated, but we have a great body of evidence, both biochemical and experimental, which puts its existence beyond doubt, and we hare the demonstration of its effects as a vivid clinical experience.

With a deranged metabolism and the imperfect catalysis which necessarily follows, further toxic elements arise. It is at this stage that the body alkalis are called upon-a last line of defence, as we have called it-and for a time it may be that this influence is sufficient to stare off disaster, but a time comes when the reserve is depleted, and when this occurs the body organization breaks down and death ensues.

Such is an outline of the picture in a serere or a neglected case. Fortunately there are wars and neans by which the tragedy can be arerted, and these we shall now discuss.

\section{Treatment.}

The inclination to focus attention upon the local lesion is one with which we can sympathize, but our judgement in this respect must not be unbalanced, lest it be the so common error of failing to see the wood for the trees. In every burn there is a general problem overshadowing in its importance the local lesion, because, if death ensues, the reason in the majority of cases is related to the general change; and the further point is true, that the local condition is the focus from which the general disturbance originates. It is apparent, therefore, that any discussion of treatment must be embodied under two heads-(A) the general problem, and (B) the local error.

\section{The General Problem.}

Shock is the state which first demands attention. It is ordinary surgical shock, in which the local injury and the psychological element both play their interacting parts. The condition is treated on the usual conventional lines: the body heat is carefully maintained and stimulants are administered; if thede is much pain, as there almost. cortainly will be, a sedative is given, and morphine is the safest and the best. If, in spite of these simple and readyto-hand measures, blood pressure readings indicate that the shock condition is deepening, fluid (gluoose saline) is given subcutaneously or by proctoclysis. The degree of shock, however, is rarely sufficiently intense to necessitate anything more than the simplest remedies.

As the shock danger is overcome a general improvement becomes manifest, and now is the opportunity to make what provision is possible against the danger that lies ahead. I have described how, in the toxaemia which to some extent must inevitably occur, special calls are ultimately made upon the body alkali reserve. It is no exaggeration to say that on the response given to this demand the issue of life or death may ultimately depend; it surely behoves us, therefore, to make all possible provision for the reckoning which is bound to come. In other words, from the moment the shock danger has abated means should be taken to alkalinize the body as completely as possible, to build up reserves of alkali sufficient, and more than sufficient, to meet the demands which will be made.

In alkaline sodium phosphate we have the best therapeutic medium for the purpose in view, and it is introduced into the body by the available routes. It is given orally (20 to 30 grains of the dibasic salt every four hours), and, being less objectionable than sodium bicarbonate, it is taken more readily by children; in a 5 per cent. solution it is given by proctoclysis. The choice falls upon alkaline sodium phosphate because it remains in the body longer than bicarbonate. For some reason the kidneys, the real avenue of its excretion, do not pass it out with the same facility as they do sodium bicarbonate. The reason may be that the alkaline sodium phosphate forms a stronger colloidal combination with the body elements, and particularly with the blood plasma.

In addition to the alkalinization, a sugar reserve is supplied, this being in view of the calls upon the liver glycogen which accompany any deterioration of that organ. The sugar is supplied in the form of glucose, as a 5 per cent. solution, and the various methods of its administration are well known to all of you. Coincident with these various preparations and precautions fluid should, of course, be supplied in abundance, preferably by the bowel and the mouth.

Is there any further precautionary measure which can be taken? One has been suggested, but I cannot speak with conviction as to its value. An attractive possibility lies in the discovery of a means by which the toxíc element in circulation could be fixed or otherwise corrected, and it has been suggested that we have such a fixative or corrective in hexamine (urotropine). This drug, when administered in solution by the bowel, is said to have a beneficial action in cases of burns, and it is further stated that the benefit springs from the corrective action of the formalin basis upon the toxic element. I understand that the idea has arisen from the fact that hexamine is occasionally used in this way in the treatment of septic toxaemias.

In these various measures, some of proved value, others empirical, we have the different preventive procedures known to us at present, and it is the duty of everyone to put them into practice when the necessity arises.

In a certain proportion of cases, in spite of all efforts, toxaemia of a severe type will develop. Are there any special means of treatment lying to our hands in this event? The late Dr. Bruce Robertson of Toronto shed some light upon this question, and, though the method may seem too specialized for use in general practice, it demands mention. When it is evident that a severe toxaemia is developing it would seem that a blood transfusion might be of benefit, partly by improving the general condition and partly by counteracting the poison in circulation. The suggestion has often been put into practice, but the results of simple transfusion have been disappointing. It was at this stage that Robertson suggested that exsanguination might be combined with transfusion, and at the present time it is in this measure that we have the only treatment likely to prove efficacious in a toxaemia of real intensity. I do not propose to enter into any detail regarding it, but the principle of its method is that the sufferer is bled in a proportion of $20 \mathrm{c.cm}$. of blood for each pound of body weight. When sufficient exsanguination has been secured, a transfusion of fresh blood from a suitable donor is given to an amount sufficient to counterbalance the loss already sustained by the exsanguination. 
The Local Error.

The local error, as the lesion "visible and seen," would seem to make the most urgent demands upon one's attention, but I hope I have succeeded in showing that it is only one point in a general problem.

We have certain ideals to set before ourselves in the local treatment of a burn, and of course I speak of the recent burn, not of the neglected septic sore-this stands in a class by itself. The ideals are to maintain asepticity of the part, to keep the affected tissues dry, and to "fix" the injured tissues in order, if passible, to prevent the dissemination of toxic products into the general circulation. The most successful local applications will be those which fulfil the above desiderata.

Before discussing the individual methods, however, there is the question of the immediate attention to the burnt area. 'To avoid suffering and to facilitate examination, a light general anaesthesia may be given. The clothes, if adherent, are removed by soaking the part in a warm sterile solution of 10 per cent. sodium bicarbonate. The way in which blisters are to be dealt with depends upon the choice of the method of local treatment. If a "fixative" or " coagulant" technique is to be followed they should be punctured and the pellicle removed, as a coagulum will not form upon detached epithelium; on the other hand, if such a "protective" technique as the paraffin method is chosen, the blisters are punctured and emptied and the pellicle allowed to remain in situ.

\section{The Types of Local Treatment.}

It is possible and convenient to group the various types of local treatment under three headings according to the ways in which they exert their individual benefits. Thus there are-

A. Fixation methods, such as the alcohol method, the tannic acid method, and others, all of which are beneficial in so far as they fix the "cooked" tissues and so arrest the production and absorption of the toxin.

B. Biochemical methods-for example, the alkaline treatment-which act by counteracting the toxic substance as it is produced.

C. Protective methods-for example, the paraffin or ambrine methods-which exert their benefits by a mechanical protective influence upon the affected tissues.

Perhaps I ought to say that there is yet a fourth method, that of excision of the burnt area. It has been seriously practised in certain Continental clinics, and it is obvious how completely it ensures the abolition of a toxaemia, but the field for its use is at best a limited one, and, in view of the success which has attended less drastic methods, it need not be considered as a practical possibility.

Permit me briefly to discuss certain individual points of the various methods which are of practical value.

\section{Fixation Methods.}

1. Treatment by Absolute Alcohol.-This method is undoubtedly one of value. It is obvious how completely it fulfils the ideals of an antiseptic, a desiccant, a fixative. Has it, then, any disadvantages? It has; its rapid evaporation makes a continuous application difficult, and, if a large area is to be treated, the expense is considerable. It finds its greatest value in localized burns of the face, because the scarring is less than by any other type of treatment. When the alcohol method is employed it is essential that the entire affected area be kept constantly moistened with the fluid. A gauze pad several layers thick is laid over the affected surface, and is kept saturated by repeated spraying or by laying a number of capillary drains upon its surface and connecting these with a small central reservoir. The method, of course, necessitates complete confinement to bed and a great deal of attention, but the results in a suitable case are remarkable.

2. The Picric Acid Method.-Until recently this was the most popular method of treatment. The composition of the fluid is as follows:

$$
\begin{array}{lllllll}
\text { Picric acid } & \ldots & \ldots & \ldots & \ldots & \ldots & 2 \text { drachms. } \\
\text { Absolute alcohol } & \ldots & \ldots & \ldots & \ldots & \ldots & 1 \frac{1}{2} \text { oz. } \\
\text { Water } & \ldots & \ldots & \ldots & \ldots & \ldots & \text { To } 2 \text { pints. }
\end{array}
$$

To obtain the best results the application should be made as a spray or as a paint, frequently repeated, and the part should be exposed to the air in order to hasten evaporation. A thin gauze covering is all that is necessary. Picrio acid is a weak antiseptic, but it has certain powers of cell fixation which may inhibit the production of toxaemia. The method is associated with a risk which must never be lost sight of ; in extensive burns the absorption may be so great that poisoning effects are produced.

3. The Aluminium Aniline Method.-This application has not met with wide recognition in this country; its use is more favoured abroad. A 2 per cent. aluminium acetate in spirit solution is mixed with a 5 per cent. alcoholic solution of methylene blue in a proportion of ten parts of the aluminium solution to one part of the methylene blue. The solution is sprayed upon the affected surface, exposure to the air is encouraged, and only a light gauze dressing is kept in place.

4. Treatment by Adrenaline.-Douglas has described promising results from the use of an adrenaline application. A solution of 1 in 10,000 is employed, frequent spray applications are made, and, as in the other methods, exposure to the air is encouraged. Adrenaline has con!siderable bactoricidal and antiseptic value, but in this instance its benefit depends upon its vaso-constrictor influence and the diminished absorption of toxin from the burnt area. Rawdin and Ferguson ${ }^{1}$ employ a solution contsisting of $1 / 2$ per cent. novocain to each ounce of which is added 10 minims of 1 in 1,000 adrenaline.

5. The Tannic Acid Treatment.-Pfeiffer first showed that the toxic extracts from burned tissues are precipitabie in vitro by mercury bichloride in an acid solution or by phosphotungstic acid. Mercury bichloride was actually used as a therapeutic measure in the treatment of burns by Fauntleroy and Ravogli, but the risks of absorption from a large burned area are too great, and the method has never met with acceptance. Davidson, while investigating phosphotungstic acid, had his attention drawn to the fact that tannic acid has similar properties with fewer disadvantages, and he introduced and elaborated its use in burns. Its value lies in the fact that it precipitates the poisonous substance in burnt tissue and tans the affected area into a resistant and partly insensitive area unlikely to be the site of any septic invasion. The application is made in various ways. After many experiments we have found the most satisfactory to be the spraying of the surface with a freshly prepared 2.5 per cent. watery solution of tannic acid every hour until the tanning is complete, as shown by the deep brown colour of the coagulum. The part is thereafter left exposed to the air, but protected from any gross injury by a sterile gauze covering. If cold is complained of, the part may be placed in an electric light cradle. When the burn is on the face, and particularly in the proximity of the eyes, 5 per cent. tannic acid ointment, made with equal parts of lanoline and vaseline as a basis, may be substituted for the aqueous solution, but the efficiency of the ointment is much less than that of the solution. In a period of time varying from fourteen to twenty days the tanned area separates, to leave a clean granulating surface. The use of tannic acid is a real advance in the treatment of burns, and more than any. other method it has reduced the mortality figure.

\section{The Biochemical Method.}

Alkaline Treatment.-Many burns do extraordinarily well if treated in a bath of warm sterile water containing 10 per cent. sodium bicarbonate, and, when a small child is severely and extensively burnt, this is a most valuable and efficient method. The child is slung in some kind of hammock arrangement, and provision is made to keep the water at a uniform temperature. If such subtotal immersion is for any reason impossible, or if the burn is small, the application of a light compress soaked in a 10 per cent. watery solution of sodium bicarbonate is soothing and efficacious. The value of the method is based on biochemical data.

Wiener $^{2}$ found that the intracellular proteoses demand $\mathbf{a}$ faintly acid medium for their full activity, and this activity is checked by exposure to an alkaline medium for any length of time. We have already alluded to certain aspects of the question in speaking of the alkalinization 
which is intended to prepare for the oncoming toxaemia, and alkalinization, both general and local, will probably figure in future schemes of burn treatment.

\section{Protective Methods.}

The Paraffin Method.-The various methods of treatment hitherto mentioned have acted either as a chemical antidote or as a fixative of cells and their toxins; the value of the paraffin method lies in the protection and mechanical fixation of the part. The original " ambrine," or No. 7 paraffin, is the best application. Its constitution is as follows :

\begin{tabular}{|c|c|c|c|c|c|c|c|}
\hline Resorcin $\quad \ldots$ & & $\cdots$ & & $\cdots$ & $\cdots$ & & \\
\hline Eucalyptus oil & $\cdots$ & $\cdots$ & ... & $\cdots$ & $\cdots$ & $\cdots$ & \\
\hline Olive oil $\ldots$ & .. & $\cdots$ & $\cdots$ & $\cdots$ & $\cdots$ & ... & 5 \\
\hline Soft paraffin & $\cdots$ & $\cdots$ & $\cdots$ & $\cdots$ & $\cdots$ & $\cdots$ & $\begin{array}{l}25 \\
67\end{array}$ \\
\hline Hard paraffin & & ... & ... & ... & $\cdots$ & & 07 \\
\hline
\end{tabular}

The combination melts at $48^{\circ} \mathrm{C}$.

There are various ways of making the application. The part may be first brushed over with absolute alcohol, and the surface thoroughly dried by a current of hot air. The burnt area is then covered with the fluid wax, which has previously been brought to the proper temperature in a water-bath. The wax is applied as a spray, or painted on with a sterile brush. Over this a thin layer of sterile cottonwool is applied, and, when the application has hardened, a further layer of wax is added, followed by an additional layer of wool, and so on until sufficient thickness has been obtained.

Souttar employs a simple and efficacious technique somewhat different from that described above. The part having been prepared with alcohol, strips of ribbon gauze one inch wide are provided. These are passed rapidly through the molten paraffin, held for an instant until the edges begin to whiten, which is the sign that the correct temperature has been reached, and then firmly applied over the affected area, the strips being laid in series edge to edge.

Whichever method is employed, the after-treatment is important. After twenty-four hours, when the application is examined, it is found that the protective covering has been raised in various places by local accumulations of serum; these are punctured with a hot metal point and evacuated. A further layer of paraffin is then added, and the process is repeated when necessary.

If the burn has been in a reasonably aseptic condition a paraffin dressing may remain in situ for ten to fourteen days or even longer. Theoretically the original dressing may remain in place until the sloughs separate; actually, reapplications are almost invariably necessary before this aim is achieved.

\section{The After-Treatment.}

While the development of granulation tissue is proceeding and healing is advancing, care must be taken to guard against the development of contraction. Its appearance is insidious, and we have yet to perfect a method for its prevention. Much, however, can be done by massage and novement and the wearing of suitable appliances.

As soon as the granulating stage is sufficiently far advanced the raw area is grafted by Thiersch grafts or pedicle grafts, but these are details not within the scope of this lecture.

The surgeon's responsibility does not cease, however, with the application of the graft. The tendency to scarring continues, and a close watch is therefore kept upon the part, and any sign of contraction is combated by such means as massage, exercises, ionization, and cautious $x$-ray therapy.

I confess it was with a measure of trepidation that $I$ chose the subject of this lecture. I dare say there are some of you who may have thought the subject a somewhat sterile one; I trust I have succeeded in dispelling any such opinion. A severe burn in a young child is one of life's catastrophes, and you and I can do much to avert disaster if we are alive to the possibilities.

\section{REPERENCRS.}

1'Annals of Surgery, February, 1925. 2 Centralbl. f. Physiol., 1905,

\section{MINERS' NYSTAGMUS.}

FREELAND EERGUS, M.D., LL.D.,

HONORARY SURGEON, EYE INYIRMARY, GLASGOW.

Is the British Medical Journal of April 30th (p. 809) there appeared a report of a contribution by Dr. Haldane to a discussion on miners' nystagmus, which discussion has now been going on in the Transactions of the Institution of Mining Engineers for over a year, and to which, through the courtesy of that institution, I contributed the opening paper; on several other occasions I had the privilege of making other remarks, the last being a reply which I made to Dr. Haldane's lecture in Glasgow on April 23rd, 1927. I will now state concisely my reasons for rejecting Dr. Haldane's defective light theory, or at any rate for holding it as not proven.

Several points demand discussion, such as the fact that the introduction of electric light has, in many pits, aggravated the disease. Then, again, his theory does not seem to explain why the disease is more frequently seen at certain periods of the year than at others. Neither Dr. Haldane nor the Nystagmus Committee takes any notice of the paroxysmal nature of the disease. A patient who has had the disease, but who has to all appearances recovered, may keep well for days or even weeks, but may then suddenly have a very severe attack. If a man has apparently recovered and is living above ground, and is not exposed again to the defective lighting, he should on Dr. Haldane's supposition keep well. As a matter of fact he is far from being immune and will certainly have subsequent attacks, some of them as severe as the first. This liability to relapse may last for years; I am now seeing cases that have been victims of the disease for five or six years. They have not been exposed to defective lighting during the whole of that time, and some of them are suffering quite acutely from the disease.

Lastly, why is nystagmus not present in all pits lit by oil safety-lamps? I am informed by a gentleman of the highest authority, Mr. G. L. Kerr, that he knows of six pits in the area in which he works in which the oil safetylamp is used, but there is no nystagmus. Is that to be explained on the supposition that the oil safety-lamps in these pits are at all times in excellent condition and that in the others they are habitually unsatisfactory? Dr. Haldane's theory does not seem to offer any explanation.

Nor has any feasible explanation been given as to why there should be, as one of the symptoms, rotational movements of the eyes. Neither Dr. Haldane nor any of his colleagues on the Nystagmus Committee has said anything about the nerve centres which must be irritated or disturbed before the rotation takes place. In neither of the reports is there any mention of disturbance of nerve centres, nor is there any discussion as to the centres likely to be involved. The two reports are extremely disappointing. I except from that criticism, however, the work done by Dr. Haldane on the chemical side. That must be taken as authoritative. The oblique muscles must be involved in any rotational action, such as that which takes place in miners' nystagmus; their action is simply secondary to the superior and inferior recti muscles. In ordinary life they have almost no independent action of their own, yet, when there is nystagmus, the movements are chiefly rotational. To me it is absolutely unthinkable that the muscles are in violent action without any disturbance of the centres supplying the nerves to these-muscles, and yet the Committee does not make any suggestions as to the nuclei and centres involved in miners' nystagmus. Because such matters are left out $I$ think that when there is another investigation a member of the committee should be a neurologist of the highest standing.

Another feature that must be taken into account is that the disease seems to appear in epidemic form. Some years it is very frequent, in others it is not nearly so common. That is a matter which, in my opinion, should be considered by persons who are in the habit of dealing with vital statistics. The twelve months preceding the coal dispute were extremely bad; so far as the area in which 